\title{
The role of vancomycin in addition with colistin and meropenem against colistin- sensitive multidrug resistant Acinetobacter baumannii causing severe infections in a Paediatric Intensive Care Unit
}

\author{
Giancarlo Ceccarelli ${ }^{1,3+}$, Alessandra Oliva ${ }^{1,3+}$, Gabriella d'Ettorre ${ }^{1,3+}$, Alessandra D'Abramo ${ }^{1,3}$, Elena Caresta ${ }^{2,3}$, \\ Caterina Silvia Barbara ${ }^{2,3}$, Maria Teresa Mascellino ${ }^{1,3}$, Paola Papoff ${ }^{2,3}$, Corrado Moretti ${ }^{2,3}$, Vincenzo Vullo ${ }^{1,3}$, \\ Paolo Visca ${ }^{4}$ and Mario Venditti ${ }^{1,3^{*}}$
}

\begin{abstract}
Background: Acinetobacter baumannii has been associated with high morbidity and mortality rates, even in pediatric patients. Therapeutic options are limited, especially when the strain is multidrug resistant.

Methods: Clinical and microbiological analyses of 4 cases of systemic infections caused by multi drug resistant A. baumannii treated with colistin/vancomycin combination at a Pediatric Intensive Care Unit were performed in order to explore the potential synergistic activity of colistin plus vancomycin. All the patients were treated with colistin, meropenem and vancomycin.

Results: Four severe infections due to MDR A. baumannii were observed. All patients treated with colistin/ vancomycin combination had a positive outcome with no infection relapses. Most importantly, no significant adverse events related to the simultaneous administration of COL plus VAN were observed. In our in-vitro experiments, the synergistic effect of the combination COL plus VAN showed an early bactericidal activity even at VAN concentration of $16 \mathrm{mg} / \mathrm{L}$, which reflects the serum trough concentrations obtained in patients.

Discussion: An antimicrobial strategy based on the activity of colistin plus vancomycin was in-vitro and in-vivo effective in life-threatening infections caused by multidrug-resistant A. baumannii in a Pediatric Intensive Care Unit, in the absence of adverse effects. Colistin plus vancomycin were highly synergic and bactericidal against carbapenem-resistant, colistin sensitive A. baumannii whereas the addition of meropenem did not enhance the in-vitro activity of colistin plus vancomycin.
\end{abstract}

Conclusions: Our results confirm existing data on the potential synergistic activity of a therapeutic strategy including colistin plus vancomycin and provide important new clinical information for its potential use as a therapeutic option against MDR A. baumannii infections, especially in the pediatric population.

Keywords: Pediatric Intensive Care Unit (PICU), Acinetobacter baumannii, Multidrug resistant gram negatives, Colistin, Vancomycin, Synergism

\footnotetext{
*Correspondence: mario.venditti@uniroma1.it

Giancarlo Ceccarelli, Alessandra Oliva and Gabriella d'Ettorre are first authors.

${ }^{\dagger}$ Equal contributors

'Department of Public Health and Infectious Diseases, University of Rome

"Sapienza", Viale del Policlinico 155, Rome, Italy

${ }^{3}$ Azienda Policlinico Umberto I, Viale del Policlinico 155, Rome, Italy

Full list of author information is available at the end of the article
}

(c) 2015 Ceccarelli et al. Open Access This article is distributed under the terms of the Creative Commons Attribution 4.0 International License (http://creativecommons.org/licenses/by/4.0/), which permits unrestricted use, distribution, and reproduction in any medium, provided you give appropriate credit to the original author(s) and the source, provide a link to the Creative Commons license, and indicate if changes were made. The Creative Commons Public Domain Dedication waiver (http://creativecommons.org/publicdomain/zero/1.0/) applies to the data made available in this article, unless otherwise stated. 


\section{Background}

Acinetobacter baumannii has been recognized as a leading nosocomial pathogen associated with significant increase of length of hospitalization and mortality rates, even in the pediatric critical care units (PICUs) [1-5]. Moreover, therapeutic options are severely limited by the emergence of strains resistant to most antibiotics including carbapenems [6].

A few epidemic lineages of $A$. baumannii have been considered to be responsible for the majority of the hospital outbreaks worldwide, primarily the three international clonal lineages (ICLs) designated as ICL-I, ICL-II and ICL-III [7]. Resistance to several antibiotics, in particular carbapenems, is the hallmark of the most successful epidemic lineages [8]. Of note, an increasing incidence of carbapenem resistance has recently been reported among multi-drug resistant (MDR) ICL-II $A$. baumannii from central Italy, with the emergence in 2009 of a major clone carrying the blaOXA-23-like determinant [9].

Nowadays, the treatment of MDR A. baumannii infections represents a real challenge. Colistin (COL)-based combinations, with or without the addition of high dose of carbapenems, have been considered the milestone of the treatment; however, in consideration of the growing rate of resistance to several antimicrobial classes, innovative approaches against this microorganism have been investigated. Among these un-orthodox combinations, COL plus glycopeptides resulted to be effective both invitro and in animal models [10-13], with the potential additional advantage of preventing the development of COL resistance in COL sensitive strains $[14,15]$.

However, in the literature there are limited and anecdotal data regarding the use of this regimen in the clinical practice, especially in the pediatric patients. In fact, previous evidences were collected only throughout retrospective analyses.

Based on the potential synergistic activity of COL plus VAN, we report the in-vitro and in-vivo effectiveness of an antimicrobial strategy consisting of COL plus VAN plus meropenem (MEM) in four cases of systemic infections caused by MDR A. baumannii in a PICU.

\section{Methods}

Over a 2-months period, four cases of systemic infections due to MDR $A$. baumannii treated with the combination colistin plus vancomycin in patients hospitalized at PICU of "Sapienza" University of Rome were observed. For each patient, clinical and microbiological data were recorded.

Written informed consent for the inclusion in this work was obtained from the patients or their legal guardians; according to local regulations, no Ethical Committee authorization is required in these cases $[16,17]$.

Isolates from clinical samples including blood, tracheal aspirate and broncoalveolar lavage underwent identification and antimicrobial susceptibility testing using the VITEK-2 (Bio-Merieux, Marcy l'Etoile, France) automated system.

Molecular analyses were performed in two strains whereas all the microbiological analyses were performed only on the case index strain.

To define the A. baumannii sequence group (SG), two multiplex PCRs designed to selectively amplify group 1 or group 2 alleles of the $\operatorname{omp} A, c s u E$ and blaOXA-51like genes were performed, and allelic profiles were interpreted according to Turton et al. [18]. The presence of four groups of OXA-carbapenemase genes (namely blaOXA-23-like, blaOXA-24-like, blaOXA-51-like and blaOXA-58-like) was detected by PCR using a multiplex assay as previously described [19, 20].

Minimal inhibitory concentrations (MICs) and logarithmic minimal bactericidal concentrations (MBClog) of MEM, COL, VAN and rifampin (RIF) were determined by broth macrodilution method (BMD) in cationadjusted Mueller Hinton broth (CAMHB) according to the Clinical Laboratory Standards Institute (CLSI) guidelines [21]. For all COL testing, a polysorbate-80 (SigmaAldrich) final concentration of $0.002 \%$ was used [22]. The MIC was defined as the lowest concentration of antibiotic that completely inhibited visible growth whereas the MBClog was defined as the lowest antimicrobial concentration which killed $\geq 99.9 \%$ of the initial bacterial count (i.e., $\geq 3 \log 10 \mathrm{CFU} / \mathrm{ml}$ ) at $24 \mathrm{~h}$.

Synergy tests were performed throughout the checkerboard method at different concentrations of the following antibiotic combinations: COL + MEM, COL + RIF, COL + VAN, MEM + VAN.

Complete synergism was defined as FIC-index (FICI) $\leq 0.5$, partial synergism as FICI $>0.5<1$, additivity as FICI $\geq 1<2$, antagonism as FICI $\geq 2$. A final inoculum of $\sim 5 \times 105 \mathrm{CFU} / \mathrm{ml}$ was used for all in-vitro experiments. In addition, the Susceptible Breakpoint Indices (SBPI) of the tested combinations was calculated [10]. To determine VAN and RIF susceptibility, the authors used breakpoints consistent with those set for Grampositive organisms by the CLSI and EUCAST [10].

Furthermore, the activity of COL, VAN and MEM, alone and in combination, was investigated by time-kill studies using an initial inoculum of $\sim 5 \times 105 \mathrm{CFU} /$ $\mathrm{mL}$. As stated before, the time kill studies were conducted only on case index strain. At 2, 4, 6, 8 and $24 \mathrm{~h}$ time points, the number of CFU was counted. The following concentrations were used for killing tests: $1 \times$ MIC COL, $1 \times$ MIC VAN, $1 \times$ MIC MEM, $0.5 \times$ MIC $\mathrm{COL}+0.25 \times \mathrm{MIC}$ VAN, $0.5 \times \mathrm{MIC} \mathrm{COL}+0.125 \times \mathrm{MIC}$ VAN, $0.5 \times \mathrm{MIC} \mathrm{COL}+0.5 \times \mathrm{MIC}$ MEM, $0.5 \times \mathrm{MIC} \mathrm{COL}$ $+0.25 \times$ MIC MEM, $0.5 \times$ MIC COL + $0.25 \times$ MIC VAN + $0.5 \times \mathrm{MIC} \mathrm{MEM}$ and $0.5 \times \mathrm{MIC} \mathrm{COL}+0.125 \times \mathrm{MIC} \mathrm{VAN}$ $+0.5 \times$ MIC MEM. 
Bactericidal activity was defined as a $\geq 3-\log 10 \mathrm{CFU} / \mathrm{mL}$ reduction of the initial bacterial count at each time point. Synergy was defined as a $\geq 2-\log 10$ decrease in CFU/mL between the combinations and its most active constituent after $24 \mathrm{~h}$. The detection limit was $10 \mathrm{CFU} / \mathrm{mL}$.

\section{Results \\ Case series}

Four severe infections [2 bloodstream infections (BSIs), 1 ventilator associated pneumonia (VAP), 1 BSI plus VAP] due to MDR A. baumannii were observed at the PICU. Clinical and microbiological data of the index case are reported in Table 1 . The index case was a newborn with a severe sepsis caused by MDR and COL sensitive $A$. baumannii. The patient was initially treated for 5 days with intravenous (i.v.) COL $(6 \mathrm{mg} / \mathrm{kg}$ equivalent to $75,000 \mathrm{IU} / \mathrm{kg}$ per day, in three divided doses following a loading dose of $6 \mathrm{mg} / \mathrm{kg}$ ) and RIF $(10 \mathrm{mg} / \mathrm{kg}$ per day). However, due to worsening of the clinical conditions, VAN (40 mg/kg per day, in three divided doses) and MEM (60 mg/kg per day, in three divided doses) were added to COL, whereas RIF was stopped because of hepatic toxicity. A prompt clinical response was observed and the patient completed a 17 days treatment course. Subsequently, the patient developed a late onset uncomplicated urinary tract infection caused by the same MDR A. baumannii (Fig. 1 and Table 1).

Based on the favourable outcome of the index case, the 3 following cases were treated with the combination COL plus VAN plus MEM. In particular, in case\#2 this strategy was started after an initial failure of the COL plus RIF treatment whereas the remaining two cases were both treated with the triple combination as initial therapy. The patients had a positive outcome with no infection relapses. Most importantly, no significant adverse events related to the simultaneous administration of COL plus VAN were observed.

\section{Microbiological studies}

Molecular analyses were performed on two clinical strains (case index and case\#2). Both isolates were PCR- positive for the blaoxa-51-like gene, confirming their identity as A. baumannii [23].

Multiplex PCRs for identification of the SG yielded the 111 allelic profiles (corresponding to SG 1 according to Turton et al. [18]), indicating that both isolates belonged to the ICL-II (Additional file 1: Figure S1).

Carbapenem resistance was associated with the presence of the blaOXA-23-like carbapenem hydrolyzing oxacillinase gene (Additional file 2: Figure S2).

According to the VITEK-2 system, the strains of the four patients were similar, and were resistant to gentamicin, ciprofloxacin, amoxicillin/clavulanate, cefotaxime, ertapenem, impenem, meropenem, trimethoprim/sulfamethoxazole, tigecycline and sensitive only to COL (MIC $=0.5 \mathrm{mg} / \mathrm{L}$ ).

Additional microbiological assays to assess the potential synergism of COL plus VAN were conducted only on the index strain.

Using the MBD method, MICs/MBCs were $1 / 1,128$ / 256, 8/128 and 128/256 mg/L for COL, MEM, RIF and VAN, respectively. A complete synergism (FIC index $<0.5$ ) was observed for COL + VAN, COL + MEM and COL + RIF whereas the combination MEM + VAN showed additivity. The SBPI values were 8.12, 16.06, 12 and 0.04 for $\mathrm{COL}+\mathrm{VAN}, \mathrm{COL}+\mathrm{MEM}, \mathrm{COL}+\mathrm{RIF}$ and MEM + VAN, respectively.

In particular, the combination $\mathrm{COL}+\mathrm{VAN}$ showed an absence of growth at the following concentrations: $0.5 \times \operatorname{MIC~COL}(0.5 \mathrm{mg} / \mathrm{L})+0.25 \times \operatorname{MIC} \operatorname{VAN}(32 \mathrm{mg} / \mathrm{L})$, $0.5 \times$ MIC COL $(0.5 \mathrm{mg} / \mathrm{L})+0.125 \times$ MIC VAN $(16 \mathrm{mg} /$ L), $0.25 \times$ MIC COL $(0.25 \mathrm{mg} / \mathrm{L})+0.25 \times$ MIC VAN $(32 \mathrm{mg} / \mathrm{L}), 0.25 \times \mathrm{MICCOL}(0.25 \mathrm{mg} / \mathrm{L})+0.125 \times \mathrm{MIC}$ VAN $(16 \mathrm{mg} / \mathrm{L})$.

The combination COL + MEM + VAN at the concentrations of $0.5 \times \mathrm{MIC}$ COL $(0.5 \mathrm{mg} / \mathrm{L})+0.5 \times \mathrm{MIC} \mathrm{MEM}$ $(64 \mathrm{mg} / \mathrm{L})+0.25 \times$ MIC VAN $(32 \mathrm{mg} / \mathrm{L})$ and $0.5 \times \mathrm{MIC}$ COL $(0.5 \mathrm{mg} / \mathrm{L})+0.5 \times$ MIC MEM $(64 \mathrm{mg} / \mathrm{L})+0.125 \times$ MIC VAN (16 mg/L) showed in-vitro activity. Given the high bacteriostatic activity of the combinations $\mathrm{COL}+$ VAN and COL + VAN + MEM, we decided to perform bactericidal analyses by using different concentration of such antimicrobials.

Table 1 Clinical characteristics and microbiological data of patients

\begin{tabular}{|c|c|c|c|c|c|c|c|}
\hline \multirow[t]{2}{*}{ Patient } & \multirow[t]{2}{*}{ Age } & \multirow[t]{2}{*}{ Underlying disease } & \multirow{2}{*}{$\begin{array}{l}\text { Diagnosis at } \\
\text { admission }\end{array}$} & \multirow{2}{*}{$\begin{array}{l}\text { Type of } \\
\text { infection }\end{array}$} & \multicolumn{2}{|l|}{ Treatment (duration, days) } & \multirow[t]{2}{*}{ Outcome $^{d}$} \\
\hline & & & & & Initial & Definitive & \\
\hline Case\#1 & $3 \mathrm{mo}^{\mathrm{a}}$ & Pierre Robin Syndrome & $\begin{array}{l}\text { Septic shock } \\
\text { (A. baumannii BSI) }\end{array}$ & BSI & $\mathrm{RIF}+\mathrm{COL}(5)$ & VAN + COL + MEM (12) & $\begin{array}{l}\text { Late relapse (UTI) } \\
\text { Cured }\end{array}$ \\
\hline Case\#2 & 21 ys & $\begin{array}{l}\text { Spastic tetraparesis; } \\
\text { dysphagia }\end{array}$ & Respiratory failure $^{b}$ & VAP \& BSI & $\mathrm{RIF}+\mathrm{COL}(5)$ & VAN + COL + MEM (15) & Cured \\
\hline Case\#3 & 25 ys & Spastic tetraparesis & Respiratory failure ${ }^{b}$ & BSI & VAN + COL + MEM (16) & $\mathrm{VAN}+\mathrm{COL}+\mathrm{MEM}^{\mathrm{C}}$ & Cured \\
\hline Case\#4 & $6 \mathrm{mo}$ & None & ARDS & VAP & VAN + COL + MEM (19) & $\mathrm{VAN}+\mathrm{COL}+\mathrm{MEM}^{\mathrm{C}}$ & Cured \\
\hline
\end{tabular}

COL colistin, VAN vancomycin, RIF rifampin, MEM meropenem, UTI urinary Tract Infection, BSI bloodstream infection, VAP Ventilator-Acquired Pneumonia, ARDS Acute Respiratory Distress Syndrome

${ }^{(a)}$ ): Case-index; $\left({ }^{b}\right)$ : caused by the underlying disease; $\left({ }^{c}\right)$ : in cases \#3-4 the initial therapy was the definitive one; $\left({ }^{d}\right)$ : at least 3-months follow-up 


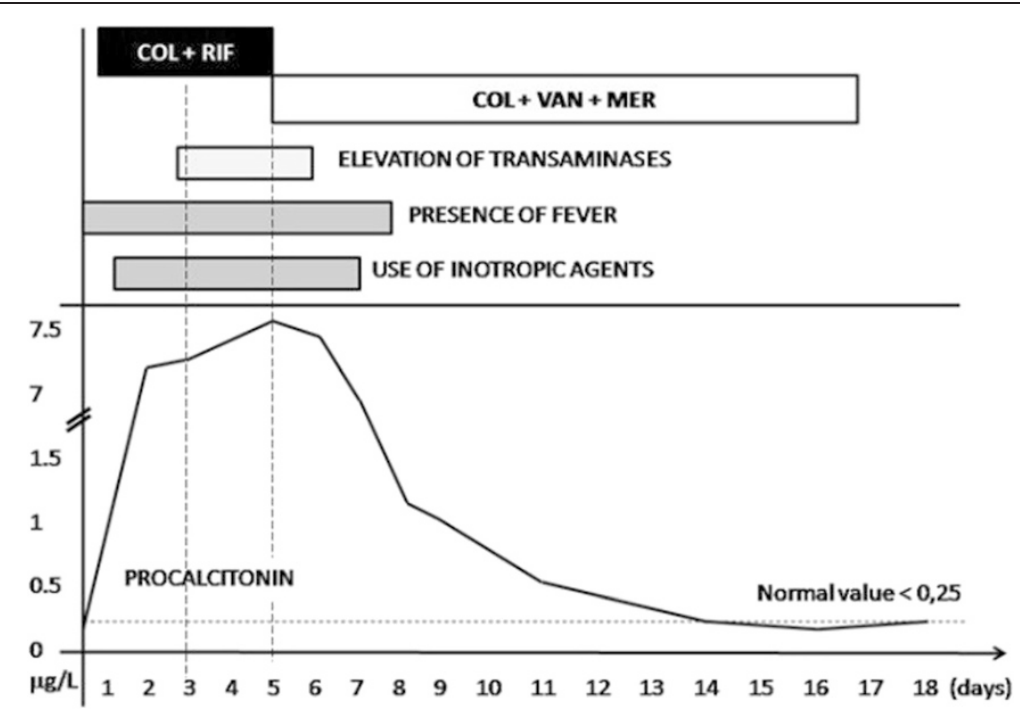

Fig. 1 Clinical and therapeutic features of case-index MDR A. baumannii bacteremia. Procalcitonin levels increased up to $7 \mathrm{mg} / \mathrm{L}$ under treatment with $C O L+R I F$, which was expression of treatment failure. Rather, it started to decrease under COL + VAN + MEM therapy and reached the normal value after 15 days of such therapy. The decreasing levels of procalcitonin following therapy with COL + VAN + MEM was expression of treatment success. COL: Colistin, VAN: Vancomycin, RIF: Rifampin, MEM: Meropenem

In the killing experiments, VAN, MEM and COL alone showed only a slight decrease of CFU/mL at $2 \mathrm{~h}, 4 \mathrm{~h}$, and $6 \mathrm{~h}$ time points; however, a significant re-growth was observed at $24 \mathrm{~h}$ for all antimicrobials (Fig. 2).

When the combination COL + VAN was tested, bactericidal activity was observed after $2 \mathrm{~h}$ at concentrations of $0.5 \times \mathrm{MIC}$ COL $(0.5 \mathrm{mg} / \mathrm{L})+0.25 \times \mathrm{MIC}$ VAN $(32 \mathrm{mg} / \mathrm{L})$ and $0.5 \times \mathrm{MIC}$ COL $(0.5 \mathrm{mg} / \mathrm{L})+0.125 \times \mathrm{MIC}$ VAN $(16 \mathrm{mg} / \mathrm{L})$, which was maintained until $24 \mathrm{~h}$ (Fig. 2a). In addition, these combinations were synergic at $24 \mathrm{~h}$. Of note, the latter combination reflects the serum trough concentrations of VAN which can be achieved during therapy $(16 \mathrm{mg} / \mathrm{L})$.

The combinations $0.5 \times \mathrm{MIC}$ COL $(0.5 \mathrm{mg} / \mathrm{L})+0.5 \times$ $\operatorname{MIC} \operatorname{MEM}(64 \mathrm{mg} / \mathrm{L})$ and $0.5 \times \operatorname{MIC} \mathrm{COL}(0.5 \mathrm{mg} / \mathrm{L})+$ $0.25 \times$ MIC MEM (32 mg/L) showed a bactericidal activity at $6 \mathrm{~h}$, which was observed until $24 \mathrm{~h}$ (Fig. 2b).

The triple combinations $0.5 \times \mathrm{MIC}$ COL $(0.5 \mathrm{mg} / \mathrm{L})$ $\mathrm{COL}+0.5 \times \mathrm{MIC} \mathrm{MEM}(64 \mathrm{mg} / \mathrm{L})+0.25 \times \mathrm{MIC} \mathrm{VAN}$ $(32 \mathrm{mg} / \mathrm{L})$ and $0.5 \times \mathrm{MIC} \mathrm{COL}(0.5 \mathrm{mg} / \mathrm{L}) \mathrm{COL}+0.5 \times$ MIC MEM (64 mg/L) + 0.125 $\times$ MIC VAN $(16 \mathrm{mg} / \mathrm{L})$ were found to be bactericidal at $6 \mathrm{~h}$, with absence of growth at $24 \mathrm{~h}$ (Fig. 2c).

\section{Discussion}

In this report, we described the in-vitro and in-vivo effectiveness of a combination containing COL, MEM and VAN against MDR $A$. baumannii causing systemic infections at a PICU.

Nowadays, the ability of $A$. baumannii to rapidly acquire antibiotic resistance has been recognized as an important therapeutic challenge. Therefore, the use of
COL has been recently reviewed and combinations of COL plus RIF or tigecycline or ampicillin-sulbactam have been used in critically ill adults and children. However, tigecycline safety and efficacy have not been established yet in pediatric patients, and its use in these subjects is so far not recommended [24-29].

The usefulness of a combination therapy has been highlighted by a recent clinical trial in patients with lifethreatening infections due to MDR A. baumannii [30]. Despite the 30-day mortality was not reduced by the addition of RIF to COL (at a daily dosage of $6 \mathrm{MUI}$ ), an increased rate of $A$. baumannii eradication was observed relative to COL alone [30].

The in-vitro synergistic activity of COL-based regimens in COL-susceptible and COL-resistant A. baumannii strains is an area of active investigation [25-29]. Among innovative approaches, the combination of COL plus other antimicrobials usually used against Gram-positive bacteria has recently gained interest. Potent in-vitro synergistic interactions between COL and daptomycin have been described, although the beneficial effects of this combination were limited only to COL susceptible isolates [31]. A study on the activity of COL plus telavancin (a novel lipoglycopeptide antibiotic with a narrow antiGram-positive spectrum) reported a marked synergism both in checkerboard and time-kill assays against COLsusceptible MDR A. baumannii isolates [32].

However, COL + VAN is regarded to as the most promising combination. A pioneer study by Gordon et al. first described an unexpected potent synergism and a sustained bactericidal activity of the VAN + COL combination against MDR COL-sensitive A. baumannii 

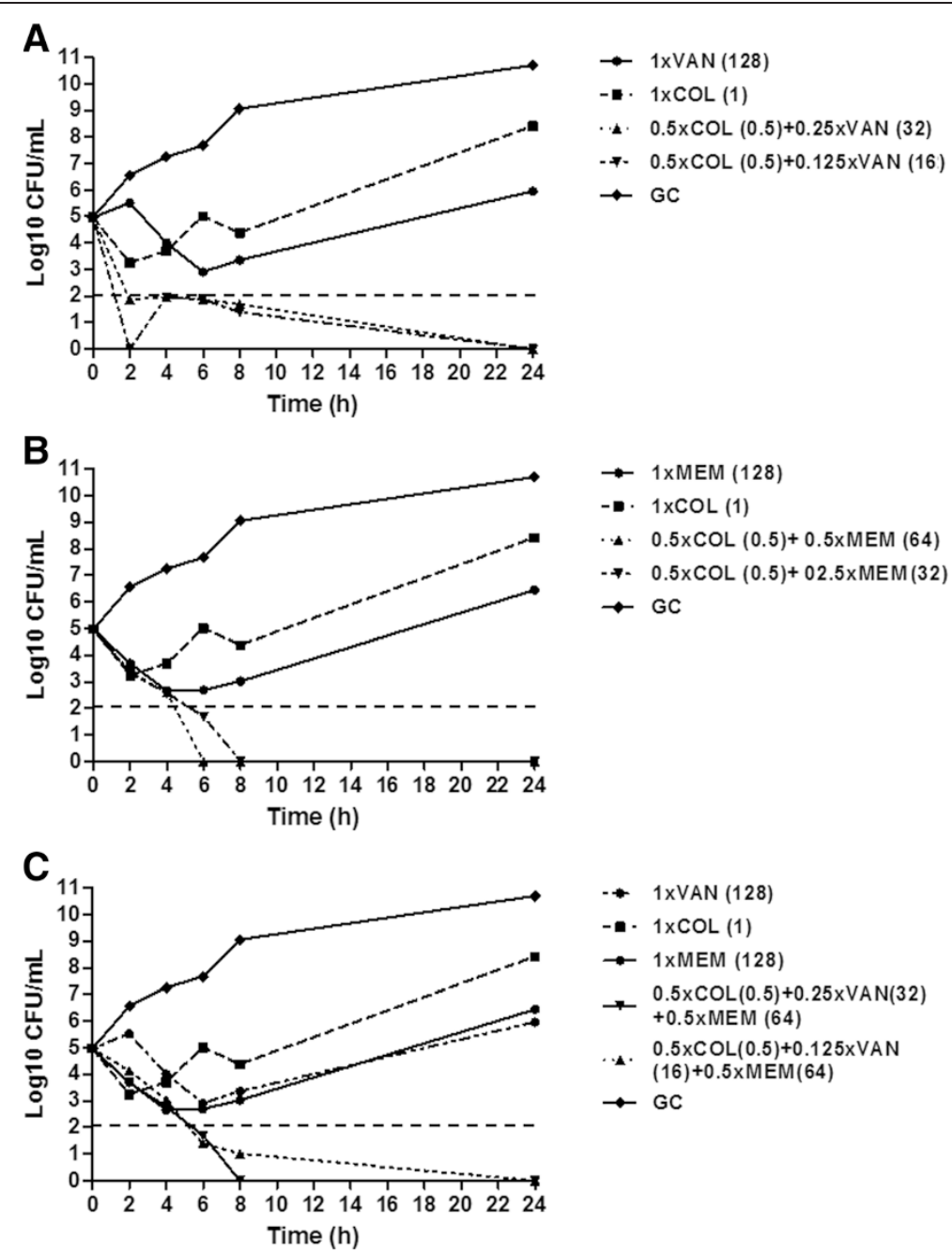

- 1 XVAN (128)

-1. 1 XCOL (1)

$\rightarrow$ 1XMEM (128)

$0.5 \times \mathrm{COL}(0.5)+0.25 \times \mathrm{VAN}(32)$

$+0.5 \times$ MEM $(64)$

$0.5 \times C O L(0.5)+0.125 \times \mathrm{VAN}$

$(16)+0.5 \times M E M(64)$

$\rightarrow$ GC

Fig. 2 Time-kill studies for colistin (COL), meropenem (MEM) and vancomycin (VAN), alone and in combination, against MDR A. baumannii. a The bactericidal activity of COL + VAN is represented. Values in bracket represent the actual concentration (mg/L). $\mathbf{b}$ The bactericidal activity of COL + MEM is represented. Values in bracket represent the actual concentration $(\mathrm{mg} / \mathrm{L})$. c The bactericidal activity of COL $+\mathrm{MEM}+\mathrm{VAN}$ is represented. The actual concentrations of the triple combinations are: $0.5 \times \mathrm{MIC} \mathrm{COL}(0.5 \mathrm{mg} / \mathrm{L}) \mathrm{COL}+0.25 \times \mathrm{MIC}$ VAN $(32 \mathrm{mg} / \mathrm{L})+0.5 \times \mathrm{MIC} \mathrm{MEM}(64 \mathrm{mg} / \mathrm{L})$ and $0.5 \times$ MIC COL $(0.5 \mathrm{mg} / \mathrm{L}) \mathrm{COL}+0.125 \times$ MIC VAN $(16 \mathrm{mg} / \mathrm{L})+0.5 \times$ MIC MEM $(64 \mathrm{mg} / \mathrm{L})$. The dashed horizontal line represents a reduction of $3 \log 10 \mathrm{cfu} / \mathrm{mL}$ compared with the initial bacterial count. GC, growth control

[10], and similar effects were later reported for COLresistant strains [12]. Interestingly, the bactericidal activity was only slightly delayed in COL-resistant compared to the susceptible isolates, suggesting that the mechanisms responsible for COL resistance in A. baumannii strains do not significantly affect the synergism of the VAN + COL combination. However, few anecdotal data on the use of this regimen in pediatric patients are available, and these were collected only through retrospective analyses [33, 34].

The rationale of using COL plus VAN resides on the hypothesis that COL increases the permeability of the outer membrane, thereby enhancing the antibacterial activity of large sized hydrophobic molecules, such as VAN, which are normally excluded by the Gram-negative outer membrane. Thus, the membrane-perturbing properties of COL could allow VAN to reach its periplasmic target at inhibitory concentrations [10]. The importance of the cell-permeabilizing properties of COL in determining synergisms with unconventional antimicrobials is consistent with the absence of in-vitro synergism observed in our study for the MEM + VAN combination.

Notably, we observed a clear clinical and microbiological response in all the patients treated with the new therapeutic approach consisting of COL plus VAN plus MEM, in the absence of adverse events. Our decision to combine VAN with COL and MEM was motivated by the lack of response to the COL plus RIF combination in the index case. This led us to suppose that a new infection was simultaneously present, and pending microbiological 
data, broad spectrum empiric therapy was started. Considering that no microorganisms other than MDR A. baumannii were documented, we were impressed by the quick clinical response of $A$. baumannii BSI to $\mathrm{COL}+$ VAN + MEM, and we wondered which role the glycopeptide and carbapenem could have had in this combination.

Clinical results were supported by in-vitro studies, showing not only a bacteriostatic synergistic activity of COL plus VAN, but also a potent bactericidal activity. These findings corroborate existing data on the synergistic activity of COL plus VAN [33, 34], thus providing novel clinical information for its potential use as a viable therapeutic option against MDR A. baumannii infections in the pediatric population.

Nevertheless, it should be pointed out that all the patients had also received MEM together with COL and VAN. Thus, the contribution of MEM to the clinical resolution of the infections should be taken into account. Time-kill results showed that the addition of MEM to COL + VAN did not enhance the bactericidal activity of the combination COL + VAN.

Interestingly, the combination of COL + VAN resulted to be more rapidly bactericidal than both COL + MEM and COL + VAN + MEM combinations. However, the combination COL + MEM showed an absence of growth at $6 \mathrm{~h}$, whereas COL + VAN, although quickly bactericidal (i.e., at $2 \mathrm{~h}$ ), still showed some bacterial growth at $6 \mathrm{~h}$ and $8 \mathrm{~h}$. The meaning of these slight differences between COL + VAN and COL + MEM observed in vitro, and their possible clinical implications, deserve further investigations.

In our in-vitro experiments, the synergistic effect of the combination COL plus VAN was impressive, with an early bactericidal activity even at VAN concentration of $16 \mathrm{mg} / \mathrm{L}$, which reflects the serum trough concentrations obtained in patients [35]. Moreover, the addition of high concentrations of MEM, which are not easily achieved in patient's serum despite prolonged infusion, did not enhance the in-vitro synergistic activity of COL + VAN. These results might be of crucial importance in the clinical practice, considering the deleterious effects that extensive use carbapenems has in the critical care setting, such as the selection of carbapenemase producing K. pneumoniae, carbapenem-resistant Pseudomonas aeruginosa and Stenotrophomonas maltophilia. In our opinion, the implementation of a therapeutic strategy aimed at treating infections caused by MDR A. baumannii based on the combination COL + VAN, without the addition of MEM, should be investigated in larger studies, in order to confirm these intriguing results in terms of clinical and microbiological effectiveness.

Although molecular analyses were performed only on two isolates, the temporal and spatial spread of infections into the PICU is suggestive of an outbreak caused by a single clone of MDR A. baumannii. In fact, both isolates belonged to the ICL-II, carried the blaOXA-23likecarbapenemase gene and showed the MDR phenotype. Moreover, they shared the typical features of the main epidemic ICL-II clone widespread in central Italy $[9,36]$. It is therefore tempting to speculate that the therapeutic protocol reported in this study would prove to be successful for the treatment of other infections caused by this worrisome A. baumannii clone, not only in the PICU setting.

Whether the COL + VAN combination could be associated with an increased risk of renal toxicity is still matter of discussion. In fact, one study reported that the rate of acute kidney injury is significantly higher in the group treated with COL plus VAN than in those receiving only COL alone [33], whereas another study reported similar nephrotoxicity in patients treated with and without a COL-glycopeptide combination [34]. In the literature, there are limited data regarding the potential nephrotoxicity of COL-based combinations in pediatric patients. However, it has been shown that only a minority of critically ill children receiving intravenous COL for MDR Gram negative infections developed renal toxicity, even when COL was co-administered with VAN [37].

\section{Conclusion}

Our study provides additional evidence that the innovative combination of COL plus VAN, together with the possible association of MEM, could represent a lifesaving therapy in selected cases of severe infections caused by MDR A. baumannii. Although our data refer to only four cases of life-threatening infections likely due to a single clone of MDR A. baumannii, it is plausible that this strategy will serve as an effective and safe therapeutic option in severe infections caused by MDR $A$. baumannii.

\section{Ethical standards}

Written informed consent to the inclusion in this work was obtained from the patients or their legal guardians.

\section{Additional files}

Additional file 1: Figure S1. Multiplex PCR analysis of ompA, CSUE and blaOXA-51-like alleles of the two A. baumannii isolates. MP1 and MP2 indicate multiplex PCRs for group 1 and 2 alleles, respectively (Turton et al. [18]). The presence of amplicons of $355 \mathrm{bp}$ (ompA), $559 \mathrm{bp}$ (blaOXA-51-like) and $702 \mathrm{bp}$ (cSUE) are typical of SG 1, corresponding to the ICL-II. M, molecular weight marker (size in base pairs). Patient 1 is the index case. (JPEG $51 \mathrm{~kb}$ )

Additional file 2: Figure S2. PCR-based detection of carbapenemase genes. For both A. baumannii isolates, the predicted amplicons of $353 \mathrm{bp}$ and 501 bp were obtained for blaOXA51-like and blaOXA-23-like genes, respectively. $M$, molecular weight marker (size in base pairs). Patient 1 is the index case. (JPEG 56 kb) 


\section{Abbreviations}

BMD: Broth macrodilution method; BSI: Blood-stream infections; CAMHB: Cation-adjusted Mueller Hinton broth; CLSI: Clinical Laboratory Standards Institute; COL: Colistin; i.v.: Intravenous; MBClog: Logarithmic minimal bactericidal concentrations; MBL: Metallo-betalactamase; MDR: Multidrug-resistant; MEM: Meropenem; MICs: Minimal inhibitory concentrations; OXA: Oxacillinases; PICU: Pediatric intensive care unit; RIF: Rifampin; VAN: Vancomycin; VAP: Ventilator associated pneumonia.

\section{Competing interest}

On behalf of all authors, the corresponding author states that there is no conflict of interest.

\section{Authors' contributions}

GC, GdE, AO took primary responsibility for the literature search, drafted the manuscript, wrote the manuscript, performed laboratory experiments, ADA, EC, CSB MTM took primary responsibility for the literature search, drafted the manuscript, performed laboratory experiments, PV performed molecular experiments, PP and CM performed acquisition and analysis of data, MV performed conception and design of the work, interpretation of data and final rewiew of the work, $W$ performed interpretation of data and final review of the work. PV, W, CM and MV critically revised the manuscript. All authors have read and approved the final version of the manuscript.

\section{Authors' information}

Not applicable.

\section{Availability of data and materials}

Not applicable.

\section{Acknowledgements}

No specific research funding was received for this project.

\section{Author details}

${ }^{1}$ Department of Public Health and Infectious Diseases, University of Rome "Sapienza", Viale del Policlinico 155, Rome, Italy. ${ }^{2}$ Pediatric Intensive Care Unit, Department of Pediatric Sciences, University of Rome "Sapienza", Viale del Policlinico 155, Rome, Italy. ${ }^{3}$ Azienda Policlinico Umberto I, Viale del Policlinico 155, Rome, Italy. ${ }^{4}$ Department of Science, Roma Tre University, Viale G. Marconi 446, Rome, Italy.

Received: 18 March 2015 Accepted: 21 September 2015

Published online: 30 September 2015

\section{References}

1. Melamed R, Greenberg D, Porat N, Karplus M, Zmora E, Golan A, et al. Successful control of an Acinetobacter baumannii outbreak in a neonatal intensive care unit. J Hosp 314 Infect. 2003;53:31-8.

2. Simhon A, Rahav G, Shazberg G, Block C, Bercovier H, Shapiro M. Acinetobacter baumannii at a tertiary-care teaching hospital in Jerusalem, Israel. J Clin Microbiol. 2001;39:389-9.

3. Ozdemir H, Kendirli T, Ergün H, Ciftçi E, Tapisiz A, Güriz H, et al. Nosocomial infections due to Acinetobacter baumannii in a pediatric intensive care unit in Turkey. Turk J Pediatr. 2011;53(3):255-60.

4. Al Jarousha AM, El Jadba AH, Al Afifi AS, El Qouqa IA. Nosocomial multidrug resistant Acinetobacter baumannii in the neonatal intensive care unit in Gaza City, Palestine. Int J Infect Dis. 2009;13(5):623-8.

5. Orsi GB, Raponi M, Franchi C, Rocco M, Mancini C, Venditti M. Surveillance and infection control in an intensive care unit. Infect Control Hosp Epidemiol. 2005;26(3):321-5.

6. Maragakis LL, Perl TM. Acinetobacter baumannii: epidemiology, antimicrobial resistance, and treatment options. Clin Infect Dis. 2008;46(8):1254-63.

7. Antunes LC, Visca P, Towner KJ. Acinetobacter baumannii: evolution of a global pathogen. Pathog Dis. 2014;71:292-301.

8. Karah N, Sundsfjord A, Towner K, Samuelsen $\varnothing$. Insights into the global molecular epidemiology of carbapenem non-susceptible clones of Acinetobacter baumannii. Drug Resist Updat. 2012;15:237-47.

9. D'Arezzo S, Principe L, Capone A, Petrosillo N, Petrucca A, Visca P. Changing carbapenemase gene pattern in an epidemic multidrug-resistant
Acinetobacter baumannii lineage causing multiple outbreaks in central Italy. J Antimicrob Chemother. 2011:66:54-61.

10. Gordon NC, Png K, Wareham DW. Potent synergy and sustained bactericidal activity of a vancomycin-colistin combination versus multidrug-resistant strains of Acinetobacter baumannii. Antimicrob Agents Chemother. 2010:54(12):5316-22

11. Hornsey M, Wareham DW. In vivo efficacy of glycopeptide-colistin combination therapies in a Galleria mellonella model of Acinetobacter baumannii infection. Antimicrob Agents Chemother. 2011;55:3534-7.

12. Vidaillac $C$, Benichou $L$, Duval RE. In vitro synergy of colistin combinations against colistin-resistant Acinetobacter baumannii, Pseudomonas aeruginosa, and Klebsiella pneumoniae isolates. Antimicrob Agents Chemother. 2012;56(9):4856-61.

13. O'Hara JA, Ambe LA, Casella LG, Townsend BM, Pelletier MR, Ernst RK, et al. Activities of vancomycin-containing regimens against colistin-resistant Acinetobacter baumannii clinical strains. Antimicrob Agents Chemother. 2013:57(5):2103-8

14. Cai Y, Li R, Liang B, Bai N, Liu Y, Wang R. In vitro antimicrobial activity and mutant prevention concentration of colistin against Acinetobacter baumannii. Antimicrob Agents Chemother. 2010;54(9):3998-9.

15. Biswas S, Brunel JM, Dubus JC, Reynaud-Gaubert M, Rolain JM. Colistin: an update on the antibiotic of the 21st century. Expert Rev Anti Infect Ther. 2012:10(8):917-34

16. AIFA Agenzia Italiana del Farmaco. http://www.agenziafarmaco.gov.it/ wsCs_render_attachment_by_id/111.285018.115401469992960af.pdf?id= 111.285023.1154014700132. Accessed 21 Sept 2015.

17. Art. 3 D.Lgs. 17 febbraio 1998, n. 23, convertito, con modificazioni, nella Legge 8 aprile 1998, n. 94.1.

18. Turton JF, Gabriel SN, Valderrey C, Kaufmann ME, Pitt TL. Use of sequence based typing and multiplex PCR to identify clonal lineages of outbreak strains of Acinetobacter baumannii. Clin Microbiol Infect. 2007;13:807-15.

19. Poirel L, Marqué S, Héritier C, Segonds C, Chabanon G, Nordmann P. OXA-58, a novel class D $\beta$-lactamase involved in resistance to carbapenems in Acinetobacter baumannii. Antimicrob Agents Chemother. 2005;49:202-8

20. Woodford N, Ellington MJ, Coelho JM, Turton JF, Ward ME, Brown S, et al. Multiplex PCR for genes encoding prevalent OXA carbapenemases in Acinetobacter spp. Int J Antimicrob Agents. 2006;27:351-3.

21. CLSI. M07-A9. Methods for dilution antimicrobial susceptibility tests for bacteria that growaerobically; approved standard. ninthth ed. Wayne, PA: Clinical and Laboratory Standards Institute; 2012.

22. Hindler JA, Humphries RM. Colistin MIC variability by method for contemporary clinical isolates of multidrug-resistant gram-negative bacilli. J Clin Microbiol. 2013;51:1678-84

23. Turton JF, Woodford N, Glover J, Yarde S, Kaufmann ME, Pitt TL. Identification of Acinetobacter baumannii by detection of the blaOXA-51-like carbapenemase gene intrinsic to this species. J Clin Microbiol. 2006;44(8):2974-6.

24. Falagas ME, Sideri G, Vouloumanou EK, Papadatos JH, Kafetzis DA Intravenous colistimethate (colistin) use in critically ill children without cystic fibrosis. Pediatr Infect Dis J. 2009;28(2):123-7.

25. Motaouakkil S, Charra B, Hachimi A, Nejmi H, Benslama A, Elmdaghri N, et al. Colistin and rifampicin in the treatment of nosocomial infections from multiresistant Acinetobacter baumannii. J Infect. 2006;53(4):274-8.

26. Karaoglan I, Zer $\mathrm{Y}$, Bosnak VK, Mete $\mathrm{AO}$, Namiduru $\mathrm{M}$. In vitro synergistic activity of colistin with tigecycline or $\beta$-lactam antibiotic/ $\beta$-lactamase inhibitor combinations against carbapenem-resistant Acinetobacter baumannii. J Int Med Res. 2013:41(6):1830-7.

27. Deveci A, Coban AY, Acicbe O, Tanyel E, Yaman G, Durupinar B. In vitro effects of sulbactam combinations with different antibiotic groups against clinical Acinetobacter baumannii isolates. J Chemother. 2012;24(5):247-52.

28. Wareham DW, Gordon NC, Hornsey M. In vitro activity of teicoplanin combined with colistin versus multidrug-resistant strains of Acinetobacter baumannii. J Antimicrob Chemother. 2011;66(5):1047-105.

29. Liang W, Liu XF, Huang J, Zhu DM, Li J, Zhang J. Activities of colistin- and minocycline-based combinations against extensive drug resistant Acinetobacter baumannii isolates from intensive care unit patients. BMC Infect Dis. 2011:11:109.

30. Durante-Mangoni E, Signoriello G, Andini R, Mattei A, De Cristoforo M, Murino $P$, et al. Colistin and rifampicin compared with colistin alone for the treatment of serious infections due to extensively drug-resistant 
Acinetobacter baumannii: a multicenter, randomized clinical trial. Clin Infect Dis. 2013;57(3):349-58.

31. Galani I, Orlandou K, Moraitou H, Petrikkos G, Souli M. Colistin/daptomycin: an unconventional antimicrobial combination synergistic in vitro against multidrug-resistant Acinetobacter baumannii. Int J Antimicrob Agents. 2014;43:370-4

32. Hornsey M, Longshaw C, Phee L, Wareham DW. In vitro activity of telavancin in combination with colistin versus Gram-negative bacterial pathogens. Antimicrob Agents Chemother. 2012;56:3080-5.

33. Garnacho-Montero J, Amaya-Villar R, Gutiérrez-Pizarraya A, Espejo-Gutiérrez de Tena E, Artero-González ML, Corcia-Palomo Y, et al. Clinical efficacy and safety of the combination of colistin plus vancomycin for the treatment of severe infections caused by carbapenem-resistant Acinetobacter baumannii. Chemotherapy. 2013:59(3):225-31.

34. Petrosillo N, Giannella M, Antonelli M, Antonini M, Barsic B, Belancic L, et al. Clinical experience of colistin glycopeptide combination in critically ill patients infected with Gram-negative bacteria. Antimicrob Agents Chemother. 2014;58(2):851-8.

35. Rybak MJ, Lomaestro BM, Rotschafer JC, Moellering RC, Craig WA, Billeter M, et al. Vancomycin therapeutic guidelines: a summary of consensus recommendations from the infectious diseases Society of America, the American Society of Health-System Pharmacists, and the Society of Infectious Diseases Pharmacists. Clin Infect Dis. 2009;49(3):325-7.

36. Minandri F, D'Arezzo S, Antunes LC, Pourcel C, Principe L, Petrosillo N, et al. Evidence of diversity among epidemiologically related carbapenemaseproducing Acinetobacter baumannii strains belonging to international clonal lineage II. J Clin Microbiol. 2012;50:590-7.

37. Kapoor K, Jajoo M, Dublish S, Dabas V, Gupta S, Manchanda V. Intravenous colistin for multidrug-resistant gram-negative infections in critically ill pediatric patients. Pediatr Crit Care Med. 2013;14(6):e268-72.

\section{Submit your next manuscript to BioMed Central and take full advantage of:}

- Convenient online submission

- Thorough peer review

- No space constraints or color figure charges

- Immediate publication on acceptance

- Inclusion in PubMed, CAS, Scopus and Google Scholar

- Research which is freely available for redistribution 